\title{
Effects of Short-Term Inhalation of Patchouli Oil on Professional Quality of Life and Stress Levels in Emergency Nurses: A Randomized Controlled Trial
}

\author{
You Kyoung Shin, $\mathrm{MS}^{1, *}$ So-Young Lee, $\mathrm{MS}_{1}^{1, *}$ Jeong-Min Lee, $\mathrm{PhD}^{2}$ \\ Purum Kang, $\mathrm{PhD}^{1,3}$ and Geun Hee Seol, $\mathrm{PhD}^{1}$
}

\begin{abstract}
Objectives: The aim of this study was to investigate the effects of patchouli (Pogostemon cablin Benth.) inhalation by emergency nurses on their stress, compassion satisfaction, compassion fatigue, burnout, blood pressure, and heart rate.

Design: A randomized controlled trial.

Setting/location: University hospital in Incheon.

Subjects: This study was performed from May to August 2018 after all subjects provided written informed consent. Fifty eligible emergency nurses were recruited and randomly allocated to inhale $5 \%$ patchouli oil in sweet almond oil (patchouli group, $n=25$ ) or pure sweet almond oil (control group, $n=25$ ).

Interventions: Nurses in the patchouli group first inhaled patchouli oil at about 10 PM (the end of an afternoon shift) and inhaled patchouli oil a second time at about 10 PM on next day (24-h interval). Nurses in the control group inhaled pure sweet almond oil following the same schedule.

Outcome measures: Outcome measured included blood pressure, heart rate, levels of stress, compassion satisfaction, compassion fatigue, and burnout.

Results: Although there were no significant differences in blood pressure, heart rate, compassion fatigue, and burnout, levels of stress were significantly lower $(0.06 \pm 0.48$ vs. $1.19 \pm 1.19, p<0.001)$ and compassion satisfaction significantly higher $(0.56 \pm 2.50$ vs. $-2.84 \pm 2.43, p<0.001)$ in the patchouli than in the control group. In addition, relative to baseline, compassion fatigue was significantly lower in the patchouli group (26.72 \pm 4.98 vs. $25.88 \pm 4.63, p=0.016)$.

Conclusions: Inhalation of patchouli oil effectively reduced the levels of stress and increased compassion satisfaction in emergency nurses, suggesting that patchouli oil inhalation may improve the professional quality of life of emergency nurses. ClinicalTrials.gov ID: KCT0004615.
\end{abstract}

Keywords: patchouli, emergency nurses, stress, compassion satisfaction

\section{Introduction}

$\mathbf{E}$ MERGENCY NURSES OFTEN encounter persons with lifethreatening physical ailments and psychologic difficulties, as well as being repetitively exposed to overcrowding, chaotic environments, trauma, and death. ${ }^{1,2}$ Long-term exposure to these situations can lead to feelings of powerlessness, confusion, isolation, and emotional exhaustion. ${ }^{3}$ If not properly managed, emergency nurses may experience symptoms such as post-traumatic stress disorder with overactive

\footnotetext{
${ }^{1}$ Department of Basic Nursing Science, College of Nursing, Korea University, Seoul, Republic of Korea.

${ }^{2}$ KT\&G Central Research Institute, Daejeon, Republic of Korea.

${ }^{3}$ Department of Nursing, College of Nursing, Woosuk University, Jeonju, Republic of Korea.

*The first two authors contributed equally to this work.
} 
sympathetic nervous system, sleep disorder, anxiety, and depression. ${ }^{4,5}$

Compassion fatigue is a series of negative emotions, such as secondary traumatic stress caused by being in contact with a subject who has experienced a traumatic event, and burnout is caused by a high workload or a nonsupportive work environment. ${ }^{6}$ Approximately $86 \%$ of emergency nurses were found to have moderate-to-high levels of compassion fatigue, and nearly $82 \%$ experienced similar levels of burnout. ${ }^{7}$ Burnout in emergency nurses showed a positive correlation with compassion fatigue and a negative correlation with compassion satisfaction. ${ }^{8}$ Moreover, moderate-to-high levels of burnout in healthcare workers were found to be closely related to poor patient safety. ${ }^{9}$ Conversely, emergency nurses may experience compassion satisfaction in caring for patients. ${ }^{10}$ Previous study reported that compassion satisfaction can act as a protective factor in the relationship between burnout and secondary traumatic stress, reducing the latter. ${ }^{11}$ Previous research indicated that nurses who were distressed and had low compassion satisfaction had significantly greater stress scores than nurses with high compassion satisfaction. ${ }^{12}$ Other research reported that preexisting stress had a negative correlation with compassion satisfaction in novice nurses. ${ }^{13}$

Compassion fatigue, compassion satisfaction, and burnout in nurses affect the professional quality of life (ProQOL) and are related to their competence. ${ }^{14}$ Strategies that increase compassion satisfaction and reduce compassion fatigue and burnout can enhance the health and the quality of care that nurses provide. ${ }^{15}$ Thus, interventions that increase compassion satisfaction and ProQOL in healthcare workers may provide significant benefits.

Patchouli (Pogostemon cablin Benth.) is a perennial aromatic herb that grows wild in South and Southeast Asia. ${ }^{16}$ Patchouli oil and patchouli alcohol, the most abundant compound in patchouli oil, ${ }^{17}$ have shown various beneficial effects in patients, including antioxidant, ${ }^{18}$ anti-inflammation, ${ }^{19}$ vasorelaxation, ${ }^{20}$ anticancer, ${ }^{21}$ and skin protection ${ }^{22}$ properties. Patchouli oil has also been shown useful for people who experience anxiety or stress ${ }^{23}$ because it can control appetite and reduce depression and stress. ${ }^{24}$ In healthy adults, patchouli oil inhalation reduced stress from a high to a medium level $^{25}$ and reduced sympathetic nerve activity by about $40 \%{ }^{26}$ Similarly, inhalation of patchouli alcohol had a sedative effect in rats, reducing their locomotor activity. ${ }^{27} \mathrm{Be}-$ cause occupational stress can contribute to burnout and ProQOL in nurses, ${ }^{28}$ patchouli oil, which is well known to reduce stress and increase relaxation in other populations, may improve the ProQOL of emergency nurses. Therefore, the present study investigated the effects of patchouli oil inhalation by emergency nurses on their self-reported stress and ProQOL, and on two objectively measured sympathetic nervous system reactions, blood pressure and heart rate.

\section{Materials and Methods}

\section{Participants}

All procedures in this study were reviewed and approved by the institutional review board of a university in Incheon, Korea (No. 1044396-201802-HR-039-01). In addition, this study was registered with CRIS.nih.go.kr (KCT0004615). Emergency nurses in a university hospital in Incheon were informed of the purposes and procedures of this study, which was performed from May to August 2018 after all subjects provided written informed consent.

The minimum number of subjects required for this study was calculated using G-power software (version 3.1). Based on a level of significance of 0.05 , a power of 0.80 , and an effect size of 0.80 , a minimum of 26 subjects in each group would be required. ${ }^{29}$ Based on a dropout rate of $15 \%$, at least 30 subjects per group were required. Therefore, 60 subjects were recruited and randomly assigned to the two groups by an investigator using a random number table. To conceal the allocation sequence, generation of the random allocation sequence and recruitment of participants were conducted by independent investigators. In addition, sweet almond oil and patchouli oil were packaged in bottles of the same shape and were sequentially numbered. Subjects were included if they (1) understood the purpose of this study and agreed to participate voluntarily, (2) had been a nurse in the emergency room for at least 6 months, (3) did not have any disease and were not being treated for illness, (4) were not pregnant, (5) did not have an abnormality in olfactory function, (6) did not have asthma or an allergic reaction to patchouli oil, (7) were not receiving stress management such as an exercise or massage program, (8) had never received aromatherapy, and (9) were not regularly taking any medication that was likely to affect mental health status.

\section{Intervention}

Patchouli oil was purchased from Aromarant Co. (Röttingen, Germany), and its composition was analyzed by gas chromatography/mass spectrometry and capillary column (HP-INNOWAX; Agilent Technologies, USA) before administration. The carrier gas was helium, maintained at a rate of $1.0 \mathrm{~mL} / \mathrm{min}$. The initial column temperature was $40^{\circ} \mathrm{C}$, increasing $3^{\circ} \mathrm{C} / \mathrm{min}$ to a maximum of $230^{\circ} \mathrm{C}$. Phytochemical compounds were identified by their relative retention time, and their identities were confirmed by comparison with reference data. The three most abundant compounds were patchouli alcohol, $\delta$-guaiene, and $\alpha$-guaiene (Table 1).

In the patchouli group, a $0.5 \mathrm{~mL}$ aliquot of $5 \%$ patchouli oil dissolved in sweet almond oil (used as solvent, control group) was dropped onto a piece of gauze, measuring $5 \mathrm{~mm} \times 10 \mathrm{~mm}$. The gauze was positioned in the philtrum. Each participant took three deep breaths and was subsequently allowed to inhale the essential oil for $20 \mathrm{~min}$ through natural breathing. The length of the study intervention was established based on the results of a previous study. ${ }^{30}$ In the control group, a $0.5 \mathrm{~mL}$ aliquot of pure sweet almond oil was dropped onto a piece of gauze, and subjects inhaled this oil in the same manner. Nurses had the first inhalation of patchouli oil at about 10 PM (the end of an afternoon shift) and the second inhalation at about 10 PM on the next day (24-h interval). To minimize stimulation and maintain a comfortable environment, all inhalations were performed in a separate conference room. All treatments were performed by the same investigator to ensure consistency of measurements. Participants were blinded to the type of essential oil they inhaled to avoid any placebo effect and were not informed of the study group to which they were allocated.

\section{Demographic measures}

All participants provided information regarding demographic characteristics (age, gender, etc.). Experience of 
Table 1. Phytochemical Compounds of Patchouli Oil DETERMINED By GaS Chromatography/Mass SPectrometry

\begin{tabular}{|c|c|c|c|}
\hline Compounds & $\begin{array}{l}\text { Retention } \\
\text { time (min) }\end{array}$ & Area & $\begin{array}{c}\% \\
\text { Area }\end{array}$ \\
\hline$\alpha$-Pinene & 19.26 & $5,564,223$ & 0.45 \\
\hline Camphene & 21.68 & 115,657 & 0.01 \\
\hline Undecane & 22.44 & 84,529 & 0.01 \\
\hline$\beta$-Pinene & 23.92 & $18,371,888$ & 1.48 \\
\hline$\beta$-Myrcene & 26.39 & 828,483 & 0.07 \\
\hline$\alpha$-Terpinene & 27.59 & 133,519 & 0.01 \\
\hline Limonene & 28.66 & $25,330,541$ & 2.04 \\
\hline 1,8-Cineole & 29.13 & 106,139 & 0.01 \\
\hline$\gamma$-Terpinene & 30.98 & $7,587,509$ & 0.61 \\
\hline 3-Octanone & 31.22 & 147,458 & 0.01 \\
\hline Cymene & 32.35 & $2,843,418$ & 0.23 \\
\hline Terpinolene & 32.93 & 246,848 & 0.02 \\
\hline 1-Octen-3-ol & 39.93 & 340,692 & 0.03 \\
\hline$\delta$-Elemene & 41.69 & $3,460,320$ & 0.28 \\
\hline Pentadecane & 41.94 & $2,122,378$ & 0.17 \\
\hline$\beta$-Patchoulene & 43.02 & $34,840,174$ & 2.81 \\
\hline Linalool & 44.23 & $6,032,243$ & 0.49 \\
\hline Linalyl acetate & 44.95 & $29,043,499$ & 2.34 \\
\hline$\alpha$-Guaiene & 47.48 & $186,499,819$ & 15.0 \\
\hline$\beta$-Caryophyllene & 48.00 & $38,574,447$ & 3.11 \\
\hline$\alpha$-Patchoulene & 50.11 & $96,641,575$ & 7.79 \\
\hline Aciphyllene & 51.95 & $32,394,773$ & 2.61 \\
\hline$\delta$-Guaiene & 52.70 & $236,226,767$ & 19.0 \\
\hline Patchoulene & 53.23 & $3,117,432$ & 0.25 \\
\hline Patchouli alcohol & 69.93 & $510,660,677$ & 41.1 \\
\hline Total & - & $1,241,315,008$ & 100 \\
\hline
\end{tabular}

traumatic events was investigated using a tool consisting of 13 items. ${ }^{10}$ Briefly, the emergency nurses were asked to determine the frequency of types of traumatic events they had experienced on duty during the previous month. Each item was rated on a five-point Likert scale $(1=$ hardly experience, $5=$ experience very often), with total scores ranging from 13 to 65 points. Higher scores were associated with a greater frequency of experience of traumatic events.

\section{Outcome measures}

The primary outcomes were compassion satisfaction, compassion fatigue, burnout (from the ProQOL); stress level; and blood pressure. The secondary outcome was heart rate. All of these variables were measured before oil inhalation and after two oil inhalations (24-h interval).

ProQOL, including compassion satisfaction, compassion fatigue, and burnout, was scored using the Korean version ${ }^{10}$ of the ProQOL compassion satisfaction/fatigue subscales, Version 5. ${ }^{31}$ This tool consists of 30 items, each rated on a five-point Likert scale ( $1=$ strongly disagree, $5=$ strongly agree). Higher scores were associated with greater levels of compassion satisfaction, compassion fatigue, and burnout. Cronbach's $\alpha$ for the experience of traumatic events was 0.90. ${ }^{10}$ For the ProQOL, Cronbach's $\alpha$ was 0.87 for compassion satisfaction, 0.80 for compassion fatigue, and 0.72 for burnout. These results indicate adequate internal consistency and construct validity and verify that the subscales of the ProQOL measure different constructs. ${ }^{32}$
Stress levels were measured using a visual analog scale, ${ }^{33}$ consisting of a $10 \mathrm{~cm}$ straight horizontal line labeled 0 (no stress) on the left side and 10 (unbearable stress) on the right side. The participants were instructed to mark the point corresponding to their levels of stress, with stress score expressed in centimeters. Higher scores were associated with greater levels of stress.

Blood pressure in the right brachial artery and heart rate were measured using an electronic sphygmomanometer (Vismo PVM 2701, Tokyo, Japan) after the participants were allowed to rest on a chair for $10 \mathrm{~min}$. Blood pressure and heart rate were each measured twice before and after oil inhalation at $5 \mathrm{~min}$ intervals, with the mean measurements before and after inhalation recorded.

\section{Data analysis}

Continuous variables are expressed as mean \pm standard deviation and categorical variables as number and percentage. The homogeneity of the two groups was tested using Mann-Whitney $U$ tests for continuous variables and Chisquare tests for categorical variables. The differences between the two groups after the intervention were analyzed by Mann-Whitney $U$ tests, whereas within group differences before and after treatment were analyzed by Wilcoxon's signed rank tests. All statistical analyses were performed using SPSS version 24.0 (IBM, USA), with $p$-values $<0.05$ considered statistically significant.

\section{Results}

\section{General characteristics of study participants}

Of the 60 subjects recruited to this study, 10 discontinued interventions and were therefore excluded. Thus, 50 subjects were included, 25 in 5\% patchouli oil (patchouli) group and 25 in sweet almond oil (control) group (Fig. 1). The general characteristics of the 50 participating emergency nurses are shown in Table 2. There were no significant differences between the groups in age, gender, body mass index, marital status, education, religion, duration of nursing career, duration of serving as emergency nurses, and experience of traumatic events.

\section{Effects of patchouli oil inhalation on outcome variables}

Table 3 shows the outcome variables (compassion satisfaction, compassion fatigue, burnout, levels of stress, blood pressure, and heart rate) in the two groups of emergency nurses before and after oil inhalation. Before inhalation of patchouli or sweet almond oil, the two groups had no significant differences in all variables. The levels of stress in the patchouli group were significantly lower after than before patchouli oil inhalation. In addition, the level of stress after inhalation was significantly lower in patchouli than in the control group. Systolic blood pressure (SBP), diastolic blood pressure, and heart rate after inhalation did not differ significantly between the control and patchouli groups.

Compassion satisfaction was significantly higher after than before patchouli oil inhalation, as well as being significantly higher in the patchouli oil group than in the control group after inhalation. Although compassion fatigue did not differ significantly between the two groups after inhalation, compassion fatigue in the patchouli group was 


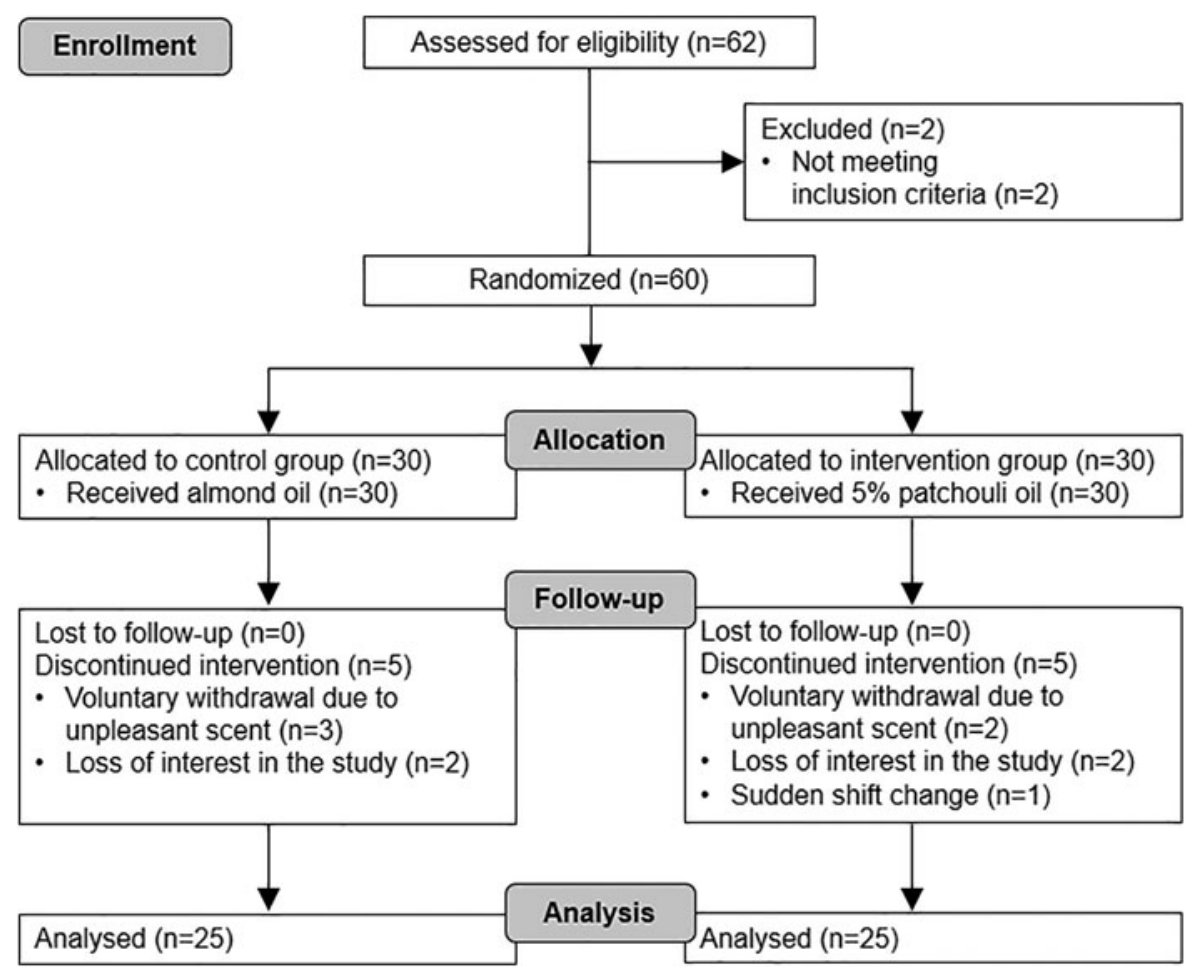

FIG. 1. CONSORT flow diagram of the study.

Table 2. Demographic and General Characteristics of Participants

\begin{tabular}{|c|c|c|c|c|c|}
\hline Variables & $\begin{array}{l}\text { Control } \\
(\mathrm{n}=25)\end{array}$ & $\begin{array}{l}\text { Patchouli } \\
(\mathrm{n}=25)\end{array}$ & $\begin{array}{l}\text { Total } \\
(\mathrm{n}=50)\end{array}$ & t or $\mathrm{X}^{2}$ & $\mathrm{p}$ \\
\hline Age (years) & $26.56(3.37)$ & $26.44(3.10)$ & $26.50(3.20)$ & -0.147 & 0.883 \\
\hline $\begin{array}{l}\text { Gender } \\
\text { Female }\end{array}$ & $25(100.0)$ & $25(100.0)$ & - & - & - \\
\hline $\begin{array}{l}\text { BMI }\left(\mathrm{kg} / \mathrm{m}^{2}\right) \\
\text { Marital status }\end{array}$ & $20.64(1.99)$ & $20.61(2.18)$ & $20.63(2.06)$ & -0.039 & 0.969 \\
\hline $\begin{array}{l}\text { Not married } \\
\text { Married }\end{array}$ & $\begin{array}{r}22(88.0) \\
3(12.0)\end{array}$ & $\begin{array}{r}22(88.0) \\
3(12.0)\end{array}$ & - & 0.000 & $1.000^{\mathrm{a}}$ \\
\hline $\begin{array}{l}\text { Education } \\
\text { Diploma } \\
\text { Bachelor or masters }\end{array}$ & $\begin{array}{r}8(32.0) \\
17(68.0)\end{array}$ & $\begin{array}{r}7(28.0) \\
18(72.0)\end{array}$ & - & 1.035 & $1.000^{\mathrm{a}}$ \\
\hline $\begin{array}{l}\text { Religion } \\
\text { Yes } \\
\text { No }\end{array}$ & $\begin{array}{r}7(28.0) \\
18(72.0)\end{array}$ & $\begin{array}{l}10(40.0) \\
15(60.0)\end{array}$ & - & 0.802 & $0.551^{\mathrm{a}}$ \\
\hline $\begin{array}{l}\text { Duration of nursing career (months) } \\
\text { Duration of nursing career in emergency unit (months) }\end{array}$ & $\begin{array}{l}43.92(29.52) \\
38.64(26.78)\end{array}$ & $\begin{array}{l}44.52(26.44) \\
41.44(26.83)\end{array}$ & $\begin{array}{l}44.22(27.74) \\
40.04(26.57)\end{array}$ & $\begin{array}{l}-0.457 \\
-0.738\end{array}$ & $\begin{array}{l}0.648 \\
0.460\end{array}$ \\
\hline Experience of traumatic events (frequency) & $34.48(6.19)$ & $36.80(5.58)$ & $35.64(5.94)$ & -1.273 & 0.203 \\
\hline Injury from motor vehicle accident & $3.44(1.00)$ & $3.68(0.95)$ & $3.56(0.97)$ & & \\
\hline Injury from a fire or explosion & $2.48(0.82)$ & $2.52(0.65)$ & $2.50(0.74)$ & & \\
\hline $\begin{array}{l}\text { Injury from a collapse } \\
\text { Injury from a machine }\end{array}$ & $\begin{array}{l}2.12(1.05) \\
3.96(0.89)\end{array}$ & $\begin{array}{l}2.20(1.04) \\
4.24(0.60)\end{array}$ & $\begin{array}{l}2.16(1.04) \\
4.10(0.76)\end{array}$ & & \\
\hline Injury from a fall & $4.40(0.65)$ & $4.44(0.65)$ & $4.42(0.64)$ & & \\
\hline Injury from a natural disaster & $1.28(0.54)$ & $1.32(0.56)$ & $1.30(0.54)$ & & \\
\hline Physical violence or abuse by family member & $2.20(0.87)$ & $2.44(0.87)$ & $2.32(0.87)$ & & \\
\hline Sexual violence or abuse by family member & $1.36(0.64)$ & $1.40(0.50)$ & $1.38(0.57)$ & & \\
\hline Physical violence or abuse by others & $4.12(0.93)$ & $4.16(1.21)$ & $4.14(1.07)$ & & \\
\hline Sexual violence or abuse by others & $1.40(0.58)$ & $1.48(0.65)$ & $1.44(0.61)$ & & \\
\hline Death from accident or homicide & $2.12(1.09)$ & $2.40(1.00)$ & $1.44(0.61)$ & & \\
\hline Injury from attempting suicide & $3.20(1.19)$ & $3.52(0.96)$ & $3.36(1.08)$ & & \\
\hline Death from committing suicide & $2.40(1.12)$ & $3.00(1.08)$ & $2.70(1.13)$ & & \\
\hline
\end{tabular}

Mann-Whitney $U$ test. Data presented as $n(\%)$ or mean (SD).

${ }^{\mathrm{a}}$ Chi-square test.

BMI, body mass index; SD, standard deviation. 
Table 3. Outcome Variables in the Control and Patchouli Groups Before AND AFTER INHALATION

\begin{tabular}{|c|c|c|c|}
\hline Variables & $\begin{array}{l}\text { Control } \\
(\mathrm{n}=25)\end{array}$ & $\begin{array}{c}\text { Patchouli } \\
(\mathrm{n}=25)\end{array}$ & $\mathrm{p}^{\mathrm{a}}$ \\
\hline \multicolumn{4}{|c|}{ Compassion satisfaction } \\
\hline Pre & $31.04(4.83)$ & $31.16(4.97)$ & \multirow[t]{3}{*}{0.800} \\
\hline Post & $30.48(4.82)$ & $34.00(4.12)$ & \\
\hline$p^{\mathrm{b}}$ & 0.300 & $<0.001$ & \\
\hline Difference & $0.56(2.50)$ & $-2.84(2.43)$ & $<0.001$ \\
\hline \multicolumn{4}{|c|}{ Compassion fatigue } \\
\hline Pre & $26.40(4.50)$ & $26.72(4.98)$ & \multirow[t]{3}{*}{0.884} \\
\hline Post & $26.48(5.10)$ & $25.88(4.63)$ & \\
\hline$p^{\mathrm{b}}$ & 0.878 & 0.016 & \\
\hline Difference & $-0.08(2.87)$ & $0.84(1.75)$ & 0.213 \\
\hline \multicolumn{4}{|l|}{ Burnout } \\
\hline Pre & $30.00(3.49)$ & $31.52(3.10)$ & \multirow[t]{3}{*}{0.123} \\
\hline Post & $30.28(3.99)$ & $30.84(3.73)$ & \\
\hline$p^{\mathrm{b}}$ & 0.645 & 0.139 & \\
\hline Difference & $-0.28(2.30)$ & $0.68(2.12)$ & 0.123 \\
\hline \multicolumn{4}{|c|}{ Stress VAS $(\mathrm{cm})$} \\
\hline Pre & $6.6(1.63)$ & $7.23(1.02)$ & \multirow[t]{3}{*}{0.132} \\
\hline Post & $6.53(1.56)$ & $6.03(1.61)$ & \\
\hline$p^{\mathrm{b}}$ & 0.054 & $<0.001$ & \\
\hline Difference & $0.06(0.48)$ & 1.19 (1.19) & $<0.001$ \\
\hline \multicolumn{4}{|l|}{ SBP (mmHg) } \\
\hline Pre & $111.56(9.05)$ & $114.02(8.18)$ & \multirow[t]{3}{*}{0.491} \\
\hline Post & $109.46(10.44)$ & $112.44(9.30)$ & \\
\hline$p^{\mathrm{b}}$ & 0.211 & 0.258 & \\
\hline Difference & $2.10(7.68)$ & $1.58(6.26)$ & 0.831 \\
\hline \multicolumn{4}{|l|}{ DBP (mmHg) } \\
\hline Pre & $73.78(6.45)$ & $75.78(8.06)$ & \multirow[t]{3}{*}{0.372} \\
\hline Post & $72.08(7.44)$ & $76.38(8.24)$ & \\
\hline$p^{\mathrm{b}}$ & 0.161 & 0.692 & \\
\hline Difference & $1.70(5.20)$ & $-0.60(6.01)$ & 0.221 \\
\hline \multicolumn{4}{|c|}{ HR (beats/min) } \\
\hline Pre & $76.26(7.62)$ & $77.54(10.36)$ & \multirow[t]{3}{*}{0.800} \\
\hline Post & $74.80(8.93)$ & $75.20(7.56)$ & \\
\hline$p^{\mathrm{b}}$ & 0.178 & 0.505 & \\
\hline Difference & $1.46(7.57)$ & $2.34(9.29)$ & 0.461 \\
\hline
\end{tabular}

Data presented as mean (SD).

${ }^{a}$ Mann-Whitney $U$ test.

'Wilcoxon's signed rank test.

DBP, diastolic blood pressure; HR, heart rate; SBP, systolic blood pressure; VAS, visual analog scale.

significantly lower after inhalation than at baseline. Burnout did not differ after inhalation in the two groups, but tended to be lower after than before patchouli oil inhalation.

\section{Discussion}

To their knowledge, this is the first study to investigate the effects of patchouli oil inhalation on stress levels, blood pressure, heart rate, and ProQOL, including compassion satisfaction, compassion fatigue, and burnout in emergency nurses. There were no significant differences in blood pressure, heart rate, compassion fatigue, and burnout between the control and patchouli groups. However, compassion fatigue was significantly reduced after inhaling patchouli oil, although there was no difference between the control and patchouli groups. In addition, levels of stress were significantly lower and compassion satisfaction significantly higher after inhaling patchouli oil than after inhaling pure sweet almond oil.

Personnel working in emergency departments are exposed to various psychosocial risk factors, including time pressure and traumatic events, and frequent hard work and low job control create occupational stresses. ${ }^{34}$ Emergency nurses also experience occupational stresses from excessive paperwork, lack of time for psychologic interventions, and high workload. ${ }^{35}$ Occupational stress plays an important role in the ProQOL and burnout of nurses. ${ }^{28}$ Surveys of emergency nurses have shown that the mean score for nurses on quality of work life was 140.15 , a moderate lev$\mathrm{el},{ }^{36}$ and the average prevalence of burnout was $26 \% .{ }^{37}$ Low levels of compassion satisfaction tended to be more frequent in emergency nurses than in nurses working in other department, ${ }^{7}$ with more than $50 \%$ of emergency nurses having a low ProQOL. ${ }^{10}$ These findings suggest the need to develop strategies enabling emergency nurses to cope with high levels of stress, compassion fatigue, and burnout and low levels of compassion satisfaction.

The 50 included nurses in this study were all women, had a mean age of 26.5 years, worked as nurses for a mean of 44.22 months, and worked as emergency nurses for a mean of 40.04 months. All of them were general nurses who were primarily involved in nursing practice, and none had a managerial position, such as head nurse. Previous studies showed that elderly nurses and nurses in managerial positions had higher compassion satisfaction. ${ }^{38}$ Thus, the participants in this study are somewhat more vulnerable to stress from various causes, and this may lower their ProQOL. Therefore, their main result that a short and simple intervention using patchouli oil effectively elevated their compassion satisfaction is an important finding.

The authors found that levels of stress were significantly lower in the patchouli group than in the control group, in agreement with results showing that patchouli oil inhalation significantly reduced levels of stress in healthy adults. ${ }^{25}$ This effect may be due to the ability of patchouli oil to reduce sympathetic nerve activity. ${ }^{26}$ Stress increases the serum concentrations of glucocorticoids and catecholamines by activating the sympathetic nervous system and the hypothalamus-pituitary-adrenal axis, increasing blood pressure and heart rate. ${ }^{39}$ A 3-month stress reduction program significantly reduced SBP and stress-related symptoms in hypertensive employees. ${ }^{40}$ However, blood pressure and heart rate did not differ significantly after inhalation of patchouli and sweet almond oil, although levels of stress were significantly lower in the patchouli than in the control group. There are several possible explanations. First, participants in this study had normal ranges of blood pressure and heart rate at baseline, so that the effects of patchouli inhalation would not be noticeable. Second, patchouli oil has a stimulating effect due to its strong fragrance, with the main constituent of patchouli oil being patchouli alcohol, which belongs to the sesquiterpene group of compounds. ${ }^{41}$ Parameters that measure the physiologic effects of aromas include blood pressure, heart rate, brain waves, electrodermal activity, and pupil function, ${ }^{42}$ with olfactory stimulation causing an immediate change in these physiologic parameters. ${ }^{43}$ Therefore, the positive effects of patchouli oil on brain function, including calm and stability, reduced stress 
levels, whereas the immediate stimulatory effects of patchouli oil did not reduce blood pressure or heart rate. ${ }^{44}$

Compassion satisfaction was found to protect subjects by reducing secondary traumatic stress, ${ }^{11}$ and increased compassion satisfaction was shown to reduce compassion fatigue and burnout. ${ }^{1}$ The authors found that compassion satisfaction was significantly higher after inhalation of patchouli oil than after inhalation of sweet almond oil. In particular, the subitems "I have happy thoughts and feelings about those I help and how I could help them.," "I am proud of what I can do to help.," and "I am happy that I chose to do this work." differed significantly between the control and patchouli groups, differences that may have been due to the calming, uplifting, and sedative effects of patchouli oil. ${ }^{44}$ Although burnout and compassion fatigue did not differ between the two groups, burnout showed a tendency to decrease and compassion fatigue was significantly lower after than before patchouli oil inhalation. Thus, short-term inhalation of patchouli oil enhanced the ProQOL of emergency nurses by reducing their stress and compassion fatigue and increasing their compassion satisfaction.

There are several limitations in the present study. First, participants inhaled patchouli oil twice on 2 days. The scent of patchouli oil lasts for up to 1 week, whereas burnout and compassion fatigue are unlikely to show immediate changes. Additional studies are needed to test the long-term effects of patchouli oil inhalation on burnout and compassion fatigue. Second, there may have been inadequate blinding to the intervention due to the specific properties of essential oils, although the authors allocated participants to two groups randomly and all participants were blinded to group assignment. To avoid bias, the duration of the intervention was relatively short, participants were not informed of the identity of the essential oil until the experiment was closed, and all interventions were conducted individually in an independent space. In future studies, a more elaborate crossover study design, in conjunction with authorized deception in consent procedures to prevent response bias, may provide a better evaluation of the effects of patchouli oil inhalation. Third, there may be potential bias in their measurements because treatments were performed by the same investigator, even though this investigator was well trained in clinical studies. Therefore, in future studies, intervention and data collection should be performed separately by different investigators. Finally, it is difficult to apply their findings to nurses in rural areas, because all of their participants were from an inner-city emergency department. Because workplace setting could influence occupational stress, there is a need for further studies that recruit nurses from more diverse regions.

\section{Conclusion}

In summary, inhalation of patchouli oil by emergency nurses reduced their stress and increased their compassion satisfaction although there were no significant differences in blood pressure, heart rate, compassion fatigue, and burnout. Inhalation of patchouli oil may be a simple and effective strategy for improving the ProQOL of emergency nurses.

\section{Acknowledgment}

This article is a revision of SYL's master thesis from Korea University.

\section{Author Disclosure Statement}

No competing financial interests exist.

\section{Funding Information}

This work was supported by a grant from the Basic Science Research Program through the National Research Foundation of Korea (NRF-2018R1D1A1B07050048) and the Institute of Nursing Research, Korea University Grant.

\section{References}

1. Flarity K, Gentry JE, Mesnikoff N. The effectiveness of an educational program on preventing and treating compassion fatigue in emergency nurses. Adv Emerg Nurs J 2013;35: 247-258.

2. Dominguez-Gomez E, Rutledge DN. Prevalence of secondary traumatic stress among emergency nurses. J Emerg Nurs 2009;35:199-204.

3. Schulte-Rüther M, Markowitsch HJ, Fink GR, Piefke M. Mirror neuron and theory of mind mechanisms involved in face-to-face interactions: A functional magnetic resonance imaging approach to empathy. J Cogn Neurosci 2007;19: 1354-1372.

4. Gentry JE, Baggerly J, Baranowsky A. Training-as-treatment: Effectiveness of the certified compassion fatigue specialist training. Int J Emerg Ment Health 2004;6:147-155.

5. Park J, Marvar PJ, Liao P, et al. Baroreflex dysfunction and augmented sympathetic nerve responses during mental stress in veterans with post-traumatic stress disorder. J Physiol 2017;595:4893-4908.

6. Stamm B. The Concise ProQOL Manual, 2nd Ed. Pocatello, ID: ProQOL.org., 2010.

7. Hooper C, Craig J, Janvrin DR, et al. Compassion satisfaction, burnout, and compassion fatigue among emergency nurses compared with nurses in other selected inpatient specialties. J Emerg Nurs 2010;36:420-427.

8. Jun Y-J, Sung MH. Effects of traumatic events, compassion fatigue, self-esteem, and compassion satisfaction on burnout of nurses in emergency department (ED). Korean J Occup Health Nurs 2014;23:80-88.

9. Hall LH, Johnson J, Watt I, et al. Healthcare staff wellbeing, burnout, and patient safety: A systematic review. PLoS One 2016;11:e0159015.

10. Kim H-J, Choi H-J. Emergency nurses' professional quality of life: Compassion satisfaction, burnout, and secondary traumatic stress. J Korean Acad Nurs Adm 2012;18:320-328.

11. Cummings C, Singer J, Hisaka R, Benuto LT. Compassion satisfaction to combat work-related burnout, vicarious trauma, and secondary traumatic stress. J Interpers Violence 2018;18:0886260518799502.

12. Hegney DG, Craigie M, Hemsworth D, et al. Compassion satisfaction, compassion fatigue, anxiety, depression and stress in registered nurses in Australia: Study 1 results. J Nurs Manag 2014;22:506-518.

13. Sprang G, Clark JJ, Whitt-Woosley A. Compassion fatigue, compassion satisfaction, and burnout: Factors impacting a professional's quality of life. J Loss Trauma 2007;12:259280.

14. Kim K, Han Y, Kwak Y, Kim JS. Professional quality of life and clinical competencies among Korean nurses. Asian Nurs Res (Korean Soc Nurs Sci) 2015;9:200-206.

15. Wang J, Okoli CTC, He H, et al. Factors associated with compassion satisfaction, burnout, and secondary traumatic 
stress among Chinese nurses in tertiary hospitals: A crosssectional study. Int J Nurs Stud 2020;102:103472.

16. Swamy MK, Sinniah UR. Patchouli (Pogostemon cablin Benth.): Botany, agrotechnology and biotechnological aspects. Ind Crops Prod 2016;87:161-176.

17. Donelian A, Carlson L, Lopes T, Machado R. Comparison of extraction of patchouli (Pogostemon cablin) essential oil with supercritical $\mathrm{CO}_{2}$ and by steam distillation. J Supercrit Fluids 2009;48:15-20.

18. Huang Q-H, Wu X, Chen X-H, et al. Patchouli oil isolated from the leaves of Pogostemon cablin ameliorates ethanolinduced acute liver injury in rats via inhibition of oxidative stress and lipid accumulation. RSC Adv 2018;8:24399 24410.

19. Li Y-C, Xian Y-F, Ip S-P, et al. Anti-inflammatory activity of patchouli alcohol isolated from Pogostemonis Herba in animal models. Fitoterapia 2011;82:1295-1301.

20. Hu G-y, Peng C, Xie X-f, et al. Patchouli alcohol isolated from Pogostemon cablin mediates endothelium-independent vasorelaxation by blockade of $\mathrm{Ca} 2+$ channels in rat isolated thoracic aorta. J Ethnopharmacol 2018;220:188-196.

21. Jeong JB, Choi J, Lou Z, et al. Patchouli alcohol, an essential oil of Pogostemon cablin, exhibits anti-tumorigenic activity in human colorectal cancer cells. Int Immunopharmacol 2013;16:184-190.

22. Lin R-F, Feng X-X, Li C-W, et al. Prevention of UV radiation-induced cutaneous photoaging in mice by topical administration of patchouli oil. J Ethnopharmacol 2014; 154:408-418.

23. Ali B, Al-Wabel NA, Shams S, et al. Essential oils used in aromatherapy: A systemic review. Asian Pac J Trop Biomed 2015;5:601-611.

24. Shankaranrayan V, Perfumery G. Patchouli constituents and its usage in perfumery. Indian Perfumer 2002;46:313 314.

25. Pujiarti R, Ohtani Y, Widowati TB, et al. Effect of Melaleuca leucadendron, Cananga odorata and Pogostemon cablin oil odors on human physiological responses. Wood Res J 2012;3:100-105.

26. Haze S, Sakai K, Gozu Y. Effects of fragrance inhalation on sympathetic activity in normal adults. Jpn J Pharmacol 2002;90:247-253.

27. Ito K, Ito M. Sedative effects of vapor inhalation of the essential oil of Microtoena patchoulii and its related compounds. J Nat Med 2011;65:336-343.

28. Wu S-Y, Li H-Y, Wang X-R, et al. A comparison of the effect of work stress on burnout and quality of life between female nurses and female doctors. Arch Environ Occup Health 2011;66:193-200.

29. Seol G-H, Jung M-H. Effect of bergamot essential oilinhalation on chronic pain after surgery for lumbar spinal stenosis. J Korean Biol Nurs Sci 2011;13:156-163.

30. Seol GH, Lee YH, Kang P, et al. Randomized controlled trial for Salvia sclarea or Lavandula angustifolia: Differential effects on blood pressure in female patients with urinary incontinence undergoing urodynamic examination. J Altern Complement Med 2013;19:664-670.
31. Stamm BH. Professional Quality of Life: Compassion Satisfaction and Fatigue Version 5 (ProQOL). Pocatello: ProQOL.org., 2009.

32. Stamm BH. The ProQOL Manual: The Professional Quality of Life Scale: Compassion Satisfaction, Burnout \& Compassion Fatigue/Secondary Trauma Scales. Baltimore, MD: Sidran, 2005.

33. Cline ME, Herman J, Shaw ER, Morton RD. Standardization of the visual analogue scale. Nurs Res 1992;41:378-380.

34. Basu S, Qayyum H, Mason S. Occupational stress in the ED: A systematic literature review. Emerg Med J 2017;34: 441-447.

35. Lu D-M, Sun N, Hong S, et al. Occupational stress and coping strategies among emergency department nurses of China. Arch Psychiat Nurs 2015;29:208-212.

36. Suleiman K, Hijazi Z, Al Kalaldeh M, Abu Sharour L. Quality of nursing work life and related factors among emergency nurses in Jordan. J Occup Health 2019;61:398-406.

37. Adriaenssens J, De Gucht V, Maes S. Determinants and prevalence of burnout in emergency nurses: A systematic review of 25 years of research. Int J Nurs Stud 2015;52: 649-661.

38. Kim SR, Kim HY, Kang JH. Effects of type D personality on compassion fatigue, burnout, compassion satisfaction, and job stress in clinical nurses. J Korean Acad Nurs Adm 2014;20:272-280.

39. Hackett RA, Steptoe A. Type 2 diabetes mellitus and psychological stress-A modifiable risk factor. Nat Rev Endocrinol 2017;13:547-560.

40. McCraty R, Atkinson M, Tomasino D. Impact of a workplace stress reduction program on blood pressure and emotional health in hypertensive employees. J Altern Complement Med 2003;9:355-369.

41. Deguerry F, Pastore L, Wu S, et al. The diverse sesquiterpene profile of patchouli, Pogostemon cablin, is correlated with a limited number of sesquiterpene synthases. Arch Biochem Biophys 2006;454:123-136.

42. Hongratanaworakit T. Physiological effects in aromatherapy. Songklanakarin J Sci Technol 2004;26:117-125.

43. Rho K-H, Han S-H, Kim K-S, Lee MS. Effects of aromatherapy massage on anxiety and self-esteem in korean elderly women: A pilot study. Int J Neurosci 2006;116: 1447-1455.

44. Perry N, Perry E. Aromatherapy in the management of psychiatric disorders. CNS Drugs 2006;20:257-280.

Address correspondence to: Geun Hee Seol, PhD

Department of Basic Nursing Science College of Nursing

Korea University

145 Anam-ro, Seongbuk-gu

Seoul 02841

Republic of Korea

E-mail: ghseol@korea.ac.kr 